\title{
Three-Dimensional Sidewall-Compression Scramjet Inlet \\ CFD Simulation and Experimental Comparison
}

\author{
Fang Chen, LiHong Chen and XinYu Chang \\ Laboratory of High Temperature Gas Dynamics \\ Institute of Mechanics, Chinese Academy of Sciences \\ Beijing, 100080, P. R. China
}

\begin{abstract}
A combination of CFD simulation and experimental comparison has been conducted for a generic three-dimensional sidewall-compression scramjet inlet configuration at Mach 5.8 with total pressure $5 \mathrm{MPa}$ and total temperature $2000 \mathrm{~K}$. The computational studies on the inlet models with four geometric contraction ratios $(C R)$ of $3,4,6$, and 8.5 were made by the three-dimensional compressible Navier-Stokes UNIC-CFD code, and have revealed the detail flow characteristics of the inner and outer flow fields. The baseline inlet model $(C R=4)$ was tested in the hypersonic propulsion test facility (HPTF), and the schlieren images of the external flow field and static pressures along the central line of walls have shown favorable comparison with CFD simulations. Also, the complete simulation results were analyzed to determine the occurrence of inlet unstart phenomena, and estimate the contraction ratio effects on the global flow features as well as performance parameters for the various configurations. From the combined computational and experimental investigation, certain recommendations for the improvements of inlet performance are provided to design and optimize the scramjet inlet configurations.
\end{abstract}

Key Words: Scramjet inlet, Contraction ratio effects

\section{NOMENCLATURE}

$C R$ : contraction ratio

$g$ : inlet throat gap

$H$ : inlet height

$L$ : distance from sidewall leading edge to throat $m_{0}$ : free stream mass flow rate

$m_{c}$ : captured mass flow rate

$M a_{\text {th }}$ : throat Mach number

$P$ : static pressure

$P_{0}$ : free stream static pressure

$P_{\text {th: }}$ : throat static pressure

$P_{t 0}$ : free stream total pressure

$P_{t, t h}$ : throat total pressure

$W$ : inlet entrance width

$X, Y$ : axial, vertical positions, respectively

$\delta$ : compression angle

$\Lambda$ : sweep angle

$\eta_{K E}:$ kinetic energy efficiency

\section{INTRODUCTION}

As a general rule, the criterion in the development of any scramjet inlet system is to find a minimum weight configuration that captures enough air mass flow to provide the required thrust over a wide range of flight and engine operation conditions with an efficient compression process, low drag, and nearly uniform flow entering the combustor. To satisfy these characteristics in different ways, a vast array of scramjet inlet concepts has been under study since the mid-1960s. In particular, extensive study has been focused on three-dimensional sidewall-compression scramjet inlet over a wide range of test conditions and geometries in Ref.1-5.

As depicted in the representative sketch of Fig.1, this genre inlet accomplishes further compression in the horizontal direction with a pair of wedge-shaped sidewalls, which greatly avoids the large-scale separation regions upstream of the inlet entrance. The cowl (bottom surface) leading edge is typically 
located aft near the throat entrance to generate a spillage flow at low speeds, thus enabling the engine with a fixed configuration to operate over a wide range of Mach numbers. Again, the wedge-shaped sidewall leading edges are aft-swept to increase the spillage flow and hence improve the inlet starting performance.

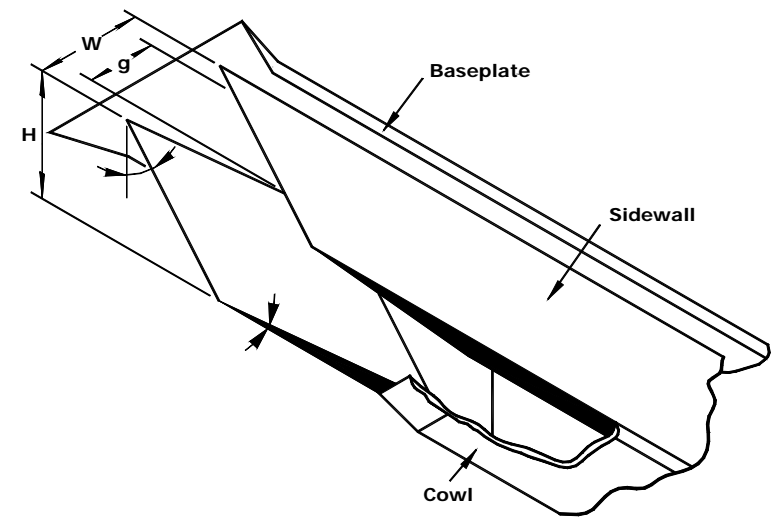

Fig. 1 Representative sketch in flight orientation

Although the typical geometry seems simple, many aerodynamic phenomena encountered in and around the inlet are very complicated, which were separately under consideration by various researchers, such as viscous phenomena, boundary-layer separation, shock/shock interaction, and shock/boundary-layer interaction ${ }^{6-11}$. It is obvious that the desired experimental data of such complicated flow features are hardly obtained with the available equipments. Therefore, a combination of CFD simulation and experimental test becomes the promising candidate, aiding in explaining the unusual or unexpected phenomena, and assessing the inlet global performances.

In the following sections, the combined computational and experimental investigation has been conducted to analyze the global flow features as well as performance parameters for various scramjet inlet configurations.

\section{CFD AND EXPERIMENTAL METHODS}

\subsection{CFD Code Description}

UNIC-CFD code $^{12}$ utilized in the computational simulation is a three-dimensional compressible Navier-Stokes flow solver, which employs the state-of-the-art unified all-speed finite volume numerical formulations with multi-block structured mesh systems, using second-order accurate numerical schemes and time marching schemes. Three-order upwind TVD scheme is used to model the convection terms and second-order central difference schemes for the viscous and source terms. To ensure some positive-definite scalar quantities such as turbulence kinetic energy and species mass fractions, a first-order upwind scheme is employed for the convection processes. A pressure based predictor/multi-corrector solution is proceeded to enhance velocity-pressure coupling and mass-conserved flow-field solutions after each time step. For steady-state applications, implicit Euler time-marching schemes are used to obtain better convergence.

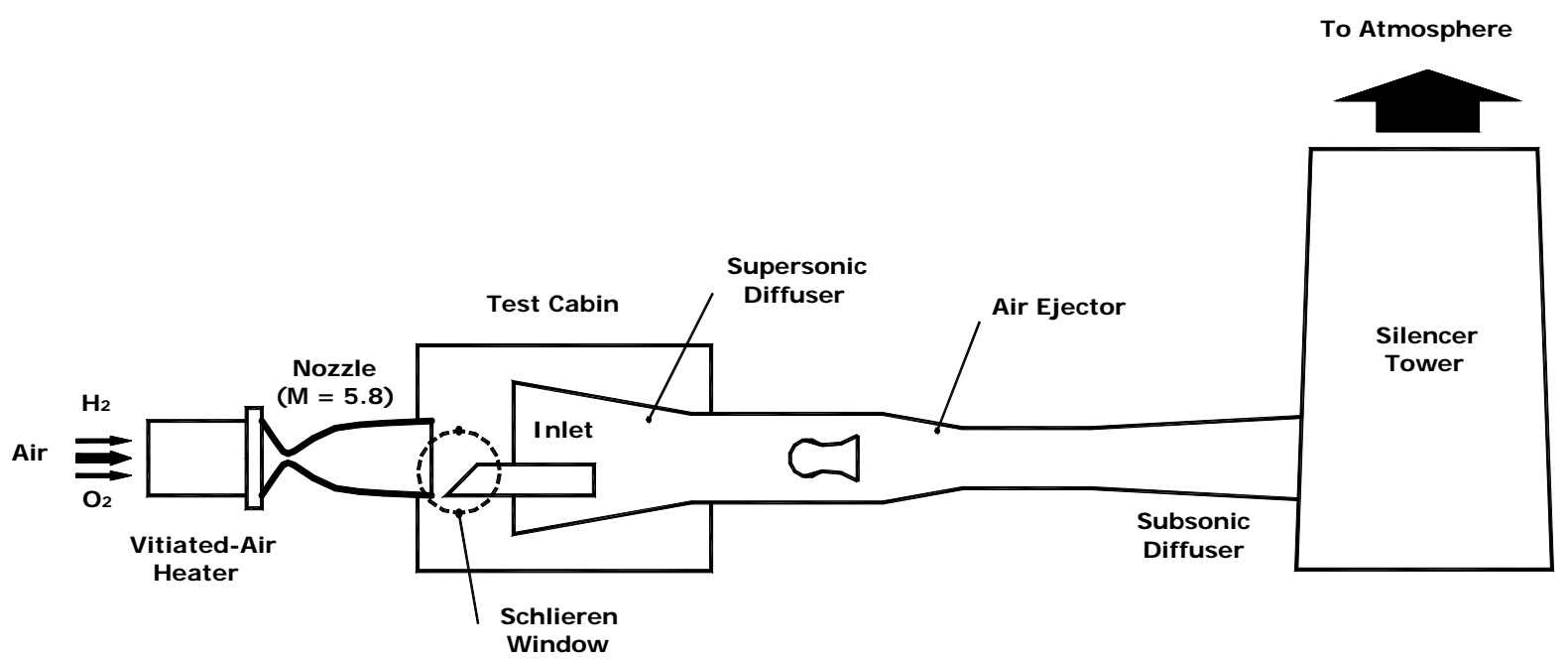

Fig. 2 Schematic of the hypersonic propulsion test facility (HPTF) 


\subsection{Computational Conditions and Experimental Apparatus}

For the CFD simulation, a design flight Mach number of 5.8 , a total pressure of $5 \mathrm{MPa}$ and a total temperature of $2000 \mathrm{~K}$, which yield a Reynolds number of approximately $5.9 \times 10^{6}$ per meter, are selected for the free stream conditions. The initial flow field conditions are identical as those obtained from the Mach 5.8 wind tunnel of HPTF ${ }^{13}$, see Fig. 2. Then the inlet model is computed with the assumed uniform inflow corresponding to the average flow field entering the inlet, and the inflow boundary is maintained laminar and fixed at free-stream conditions.

As depicted as Figure 2, the test cabin holds a schlieren window, and then schlieren images of the external flow field are given to verify starting operation of the tunnel and determine the occurrence of inlet unstart phenomena. Again, static pressures along the central line of walls are measured to record the inlet compression process.

\subsection{Inlet Models and CFD Simulations}

As shown in Fig. 3, the baseline inlet model is composed of a pair of sidewalls, a baseplate (top plane), and a cowl. The sidewalls are $70 \mathrm{~mm}$ in height and $774 \mathrm{~mm}$ in total length. The tip angle of
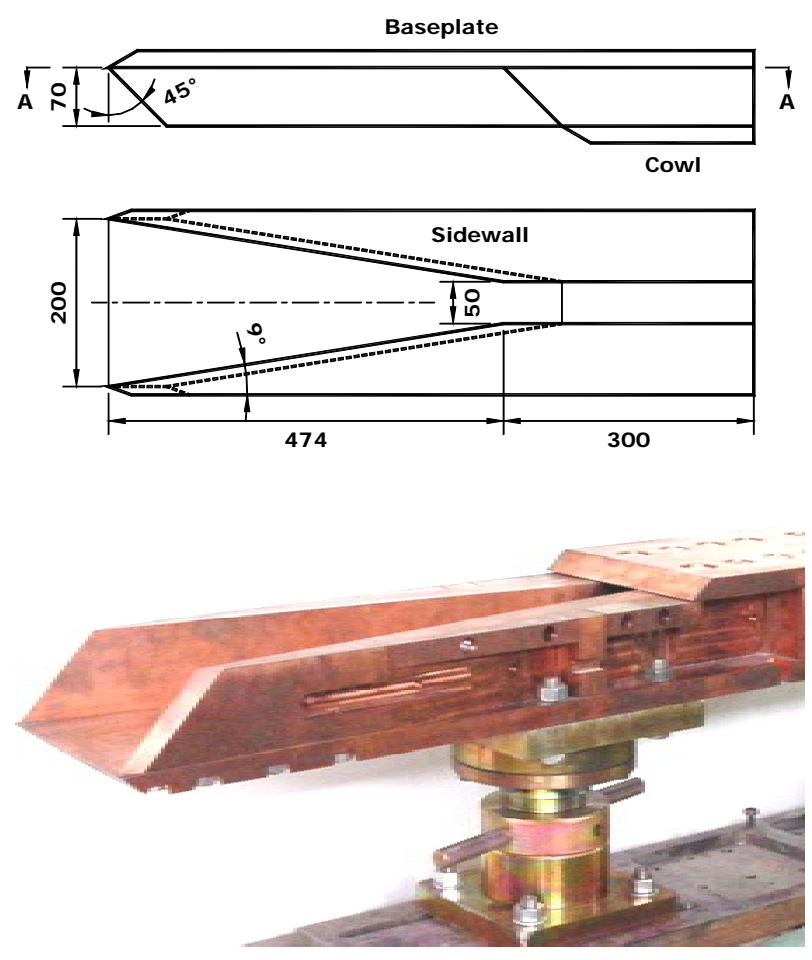

Fig.3 Sketch and photo of baseline inlet model wedge-shaped sidewalls is $9^{\circ}$ and $C R$ is 4 , which determine the length of compression section from the sidewall leading edge to the throat entrance. Herein the sidewalls are aft-swept with the same angle of $45^{\circ}$. In the present work, translating the fixed-shape sidewalls close or far, various distance between the pair were obtained, so that the four inlet models with $C R$ of $3,4,6$, and 8.5 are under consideration.

Since the inlet configuration is symmetric, only half is modeled. The internal computational grid systems, as shown in Fig. 4, have 309 grid points in the axial direction, 55 vertically, and 35 laterally to capture the whole regions. A biased clustering of the node points is appropriately utilized at the leading edge of baseplate, cowl, and sidewalls to satisfy anticipated flow gradient requirements such as shock and boundary layer, etc.

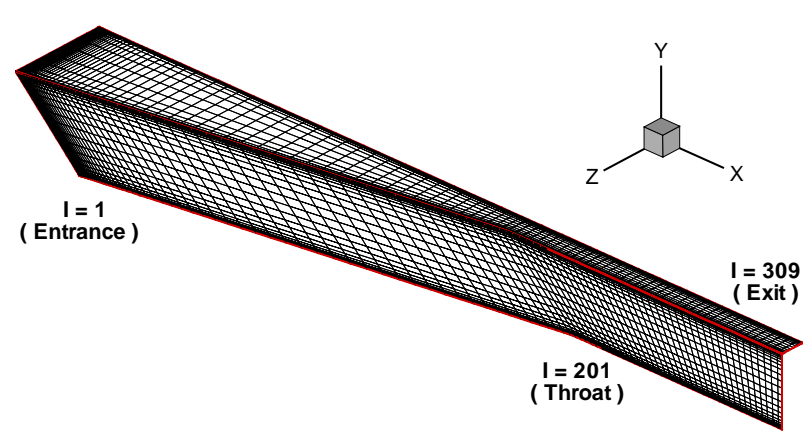

Fig. 4 Internal computational grid systems

\section{Results AND Discussions}

\subsection{Flow Fields of Baseline Inlet}

Contours of compression ratio $\left(P / P_{0}\right)$ on the baseline inlet surfaces obtained by CFD simulation are presented in Figs. 5 (a), and (b). The baseplate boundary layer is noted to develop along the baseplate centerline, which formed the weak compression in the vertical direction. The first shock wave is observed to generate from the sidewall leading edge, intersect with the opposite shock wave, and then impinges on the sidewall near the throat entrance (shoulder). Also, the figures present that the downward flow is spilled obviously out of the open bottom, and such spillage flow strongly impinges the cowl plane, thus emanating the shock wave from the cowl lip.

$P / P_{0}$ contours in twelve cross-section planes are shown in Fig. 5 (c), which track the internal 
compression in the axial direction. It is important to note that the sidewall shock waves almost intersect at $X / L=0.71(I=160)$, and impinge at the shoulder $(I=$ 201). After the later plane, the emanated cowl shock wave interacts with the sidewall shock wave in the vicinity of bottom surface, and the borne interaction zone tends to dominate the cross-section till the exit plane.

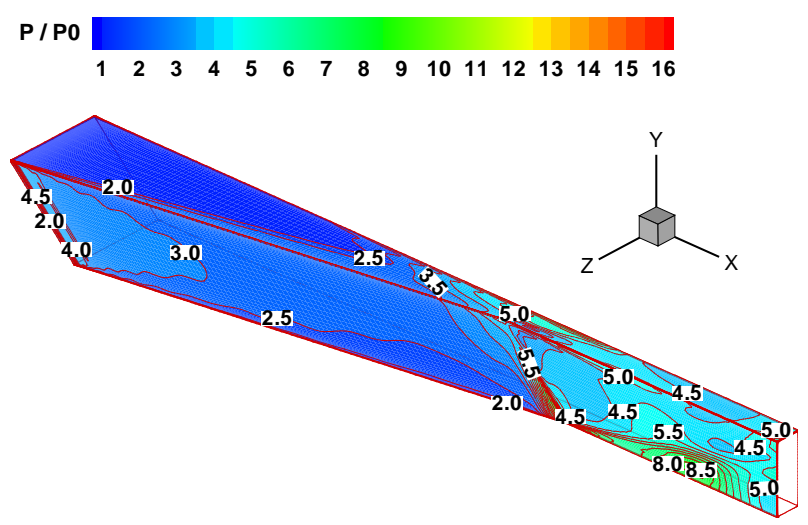

Fig. 5 (a) $P / P_{0}$ contours on baseplate $\&$ sidewall

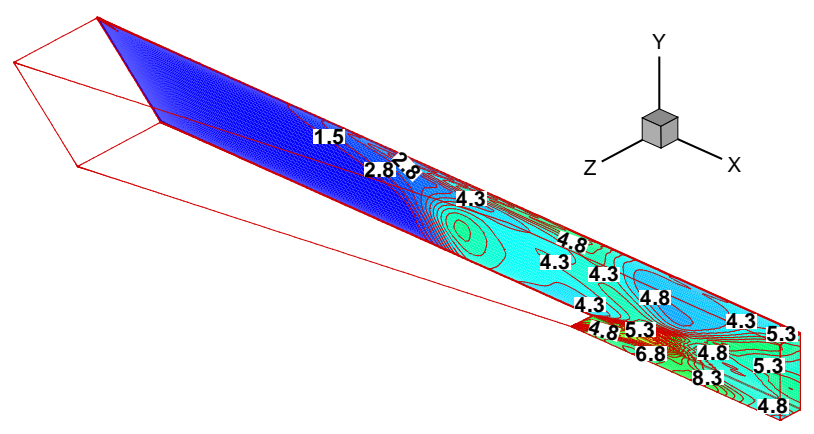

Fig. 5 (b) $P / P_{0}$ contours on symmetry \& cowl

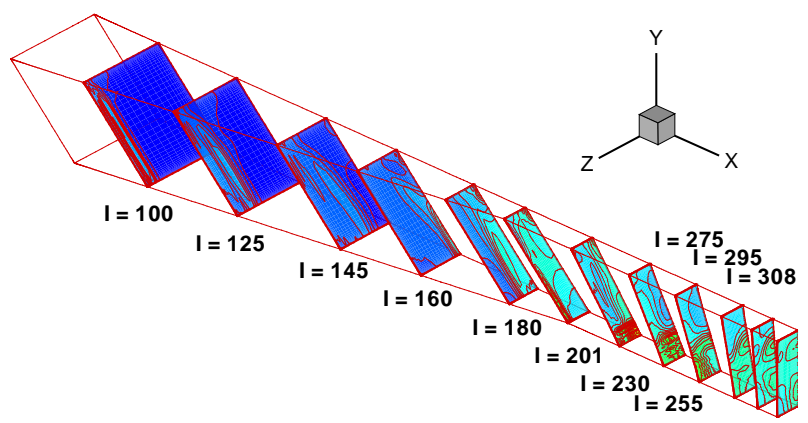

Fig. 5 (c) $P / P_{0}$ contours on cross-section planes

Figs. 6 (a), (b), and (c) show the $P / P_{0}$ contours at three heights within the inlet $(Y / H=0.25,0.50$, and 0.75 ), respectively. It is noted that the first sidewall shock wave is nearly unchanged with the different heights, but more reflect shock waves appear inside the throat at higher vertical position. In addition, the growing baseplate boundary layer is observed to distort the sidewall shock wave at $Y / H=$
0.75 , near the baseplate.

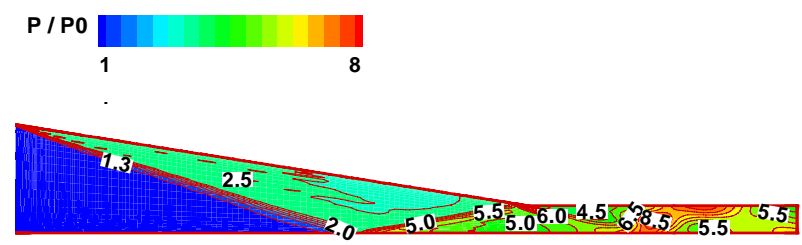

Fig. 6 (a) $P / P_{0}$ contours at $Y / H=0.25$

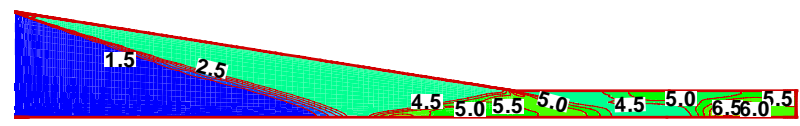

Fig. 6 (b) $P / P_{0}$ contours at $Y / H=0.50$

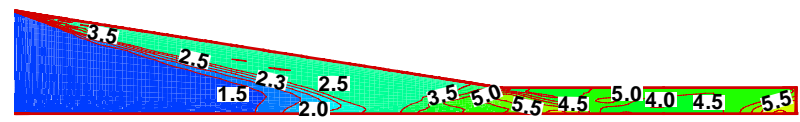

Fig. 6 (c) $P / P_{0}$ contours at $Y / H=0.75$
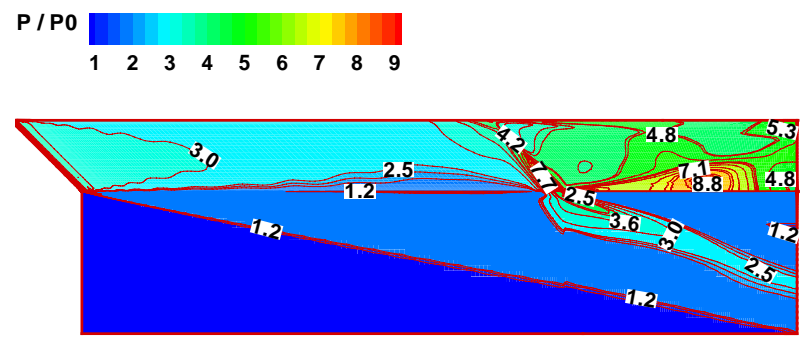

Fig. 7 (a) Side views of $P / P_{0}$ contours on sidewall

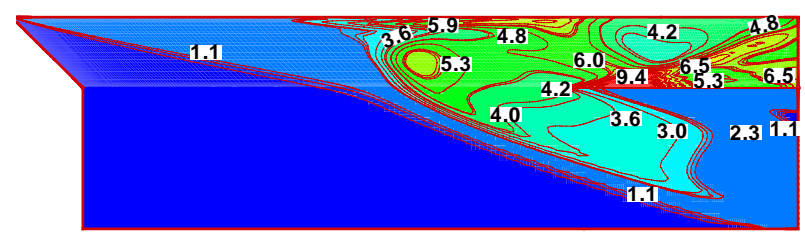

Fig. 7 (b) $P / P_{0}$ contours on symmetry plane

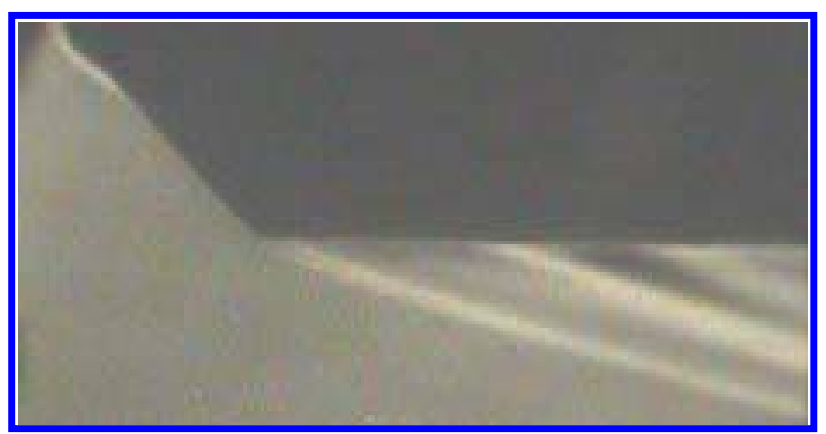

Fig. 7 (c) Schlieren photo of baseline inlet model

The side views of $P / P_{0}$ contours on the sidewall and symmetry plane obtained by CFD are respectively shown in Figs. 7 (a) and (b). Also, the flow field around the baseline inlet is presented in the schlieren photo of Fig. 7 (c). In comparison, the sidewall shock waves emanated from the sidewall leading edges and the downward flow spilled out of 
the inlet model are well captured in Figs. 7 (a) and (b). Moreover, with these side views of the overall flow field, the effects of the shock waves and the spillage flow can be seen in detail.

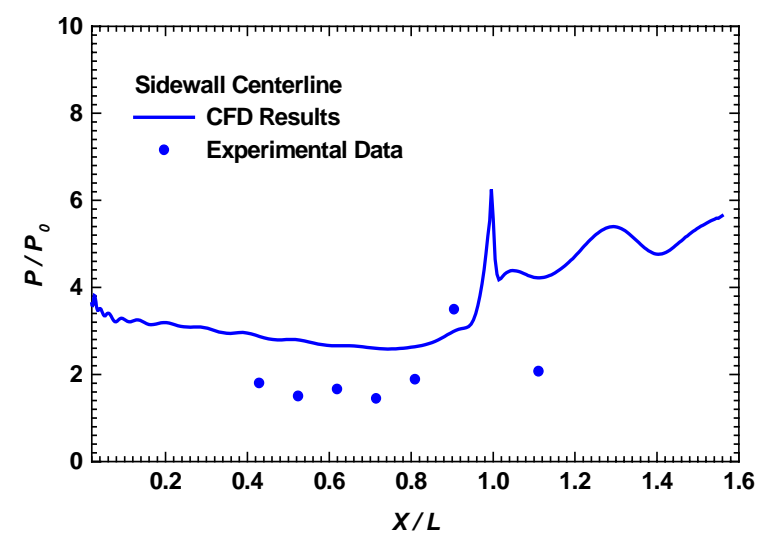

Fig. 8 Sidewall centerline $P / P_{0}$ distributions

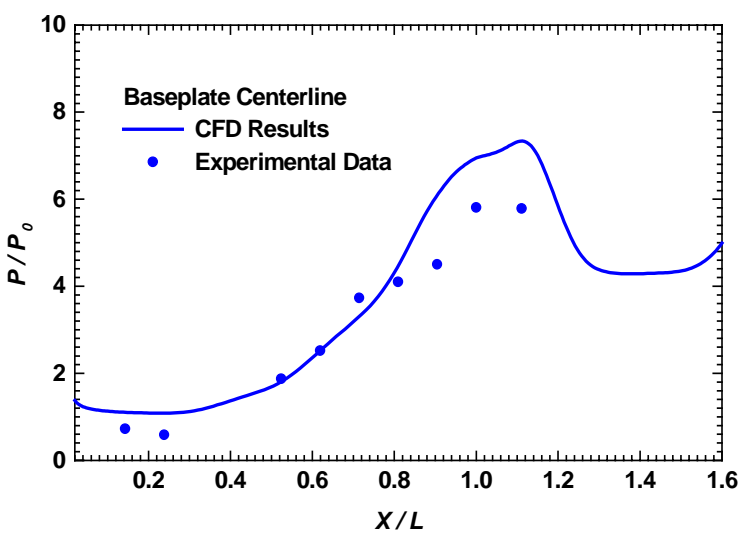

Fig. 9 Baseplate centerline $P / P_{0}$ distributions

Fig. 8 presents sidewall centerline $P / P_{0}$ distributions. Combined with the $P / P_{0}$ contours at $Y /$ $H=0.50$ in Fig. 6 (b), it is important to note that: A large pressure value initially appears at the sidewall leading edge, which represents the static pressure downstream of leading edge shock. Then the viscous interaction causes sidewall compression to decrease monotonically. Subsequently, static pressure strongly increases due to the reflected shock impingement, resulting in the upstream gradual pressure rise. After the pressure peak at the throat entrance, another drop comes forth due to evident flow expansion around the shoulder. Eventually, another pressure rise aft is observed because of the next shock impingements. Therefore, corresponding pressure relief is observed.

Fig. 9 presents baseplate centerline $P / P_{0}$ distributions. The progressive pressure rise is observed nearly $X / L=0.25$, and indicates the downward airflow departed from the baseplate. And then the expansion around the shoulder gradually brings the pressure decline. Following the pressure trough, the distribution curve climbs up again due to the next sidewall shock impingement.

In comparison with the simulation results, as shown in Figs 8 and 9, the wall static pressure measurements taken along the same lines are plotted with scatter of points. Although the sidewall centerline pressure distributions are overpredicted and the peak of the measurements is not captured (Fig.8), the rapid pressure rise and decline show a quantitative agreement in the correct locations. However, the baseplate centerline pressure shows an extremely close fit (Fig. 9), while the magnitudes downstream of the location $X / L=0.80$ are slightly overpredicted again. Therefore, the CFD simulation demonstrates reasonably good agreement with the experimental data in predicting the static pressure of the walls.

\subsection{Contraction Ratio Effects}

\subsubsection{Pressure Distributions and Contours}
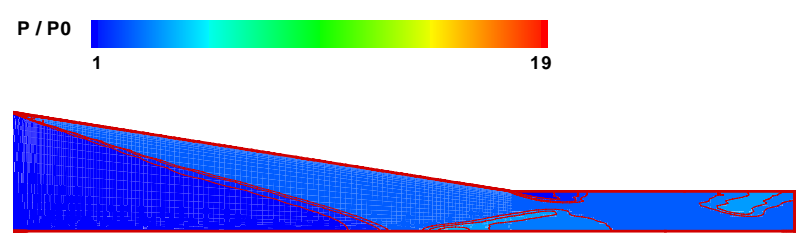

$C R=3$

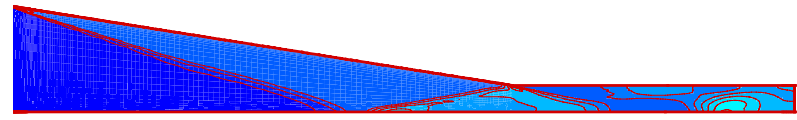

$C R=4$

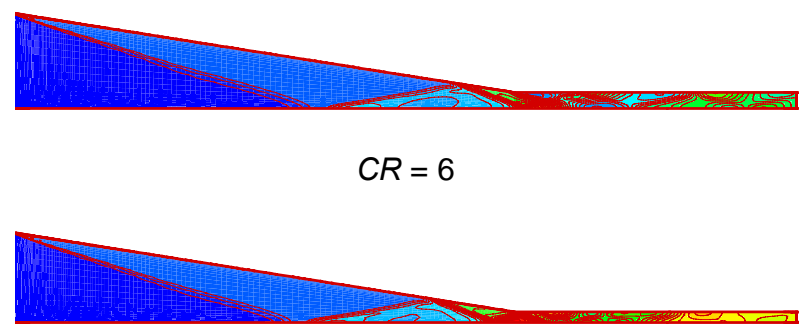

$C R=8.5$

Fig. $10 P / P_{0}$ contours at $Y / H=0.50$

For various $C R$ of $3,4,6$, and 8.5, contours of $P$ / $P_{0}$ at $Y / H=0.50$ are presented in Fig. 10. Obviously, 
the impingement location of induced sidewall shock wave varies as a function of $C R$. As $C R$ gradually increases, the intersection and impingement locations move upstream. Therefore, more reflections occur, especially accumulated in the throat, which would further strengthen internal compression.

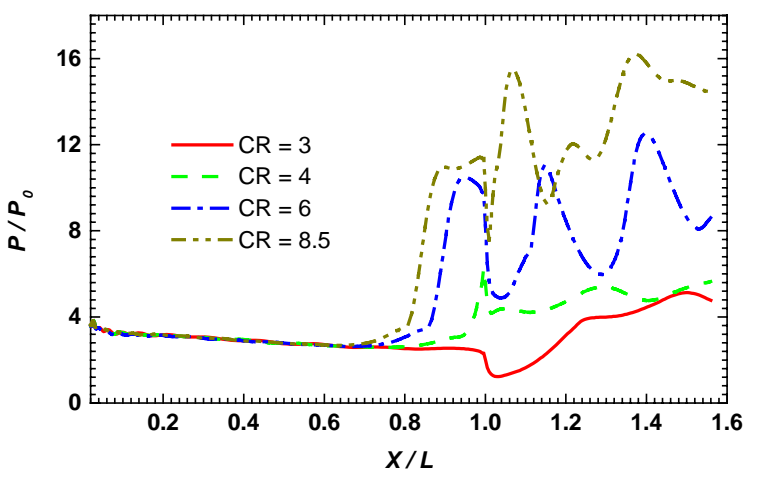

Fig. 11 (a) Sidewall centerline $P / P_{0}$ distributions

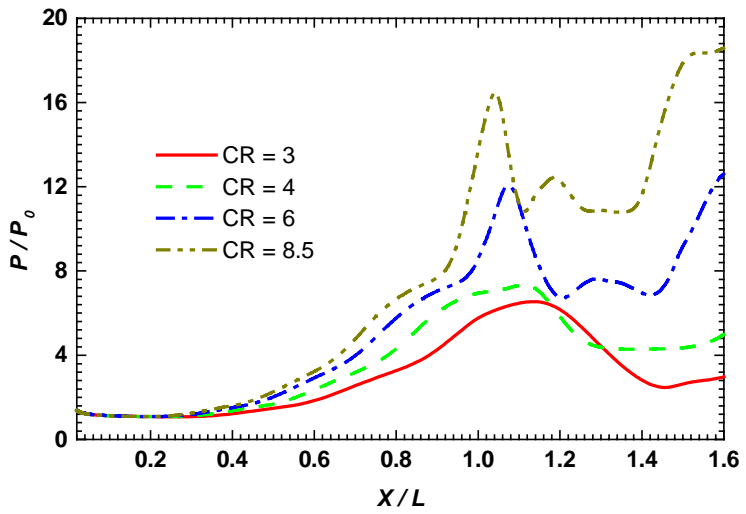

Fig. 11 (b) Baseplate centerline $P / P_{0}$ distributions

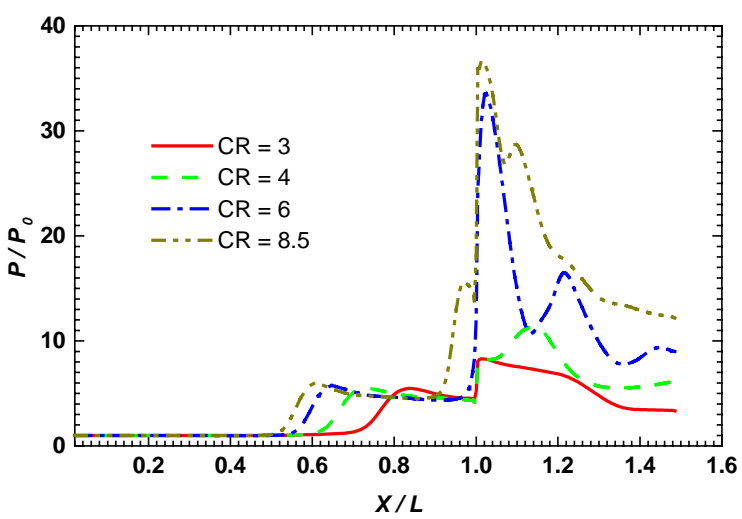

Fig. 11 (c) Cowl centerline $P / P_{0}$ distributions

More details of this salient feature are observed in sidewall centerline $P / P_{0}$ distributions, shown in Fig. 11 (a). As aforementioned, sidewall compression process can be explicitly divided into several similar steps. However, for $C R$ of 3 , near the shoulder, the compression is observed to decline considerably, even lower than the value compressed before. It indicates that reflected shocks encounter the sidewall far downstream of the throat entrance, and then the expansion around the shoulder severely cuts down the compression. Whereas for higher $C R$ of 6 and 8.5, the pressure rise moves farther upstream of the throat with increasing $C R$, which represents shock impingements have been pushed upstream. Again, the strong pressure rise and decline appears approximately twice in the throat. This sawtooth pattern means that the reflected shocks continually hit the sidewall till the exit plane.

The baseplate centerline $P / P_{0}$ distributions are presented in Fig. 11 (b). The progressive baseplate pressure rise is observed nearly $X / L=0.25$, and indicates the departure airflow from the baseplate. And then the expansion around the shoulder gradually brings the pressure decline. Following the pressure trough, the distribution curve climbs up again due to the next sidewall shock impingement. Therefore, the starting points of pressure rise are pushed upstream and the magnitudes increase with higher $C R$, which is associated with the shock impingements mentioned before.

The cowl centerline $P / P_{0}$ distributions, as shown in Fig. 11 (c), indicate that the pressure on the cowl primarily depends on the strong cowl shock wave. It can be clearly demonstrated in Fig. 12, internal $P / P_{0}$ contours on symmetry plane, which present that the airflow turns downward and impinges at the cowl lip, thus developing a strong shock wave inside the inlet. A sharp pressure rise is therefore observed at the throat in Fig. 11 (c). It can be also seen that $C R$ progressive increase strengthens the airflow downward tendency, which increasingly intensifies the cowl shock wave. For $C R$ of 8.5 , the pressure explodes to a maximum pressure of 37 , and propagate upstream of the throat entrance. Then, the inlet can hardly start for the given conditions. More details of unstart phenomena will be discussed in the following section. 


\subsubsection{Inlet Unstart}

For proper operation scramjet inlets must operate in a started mode. Extensive study has been devoted to find that inlet starting is primarily influenced by $C R$. Therefore, it is crucial to identify the starting limits of $C R$. Trexler, Auslender, and Weidner (Ref. 14) presented that the flow field of an unstart inlet is usually characterized by an strong shock wave that expelled from the inlet throat station to a station just upstream of the cowl lip. That is, the unstart inlet manifests itself by a sudden increase in static pressure on the cowl surface upstream of the cowl lip.
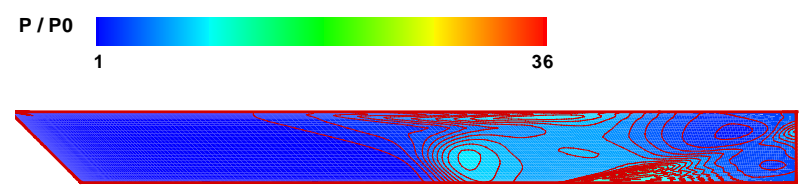

$C R=3$

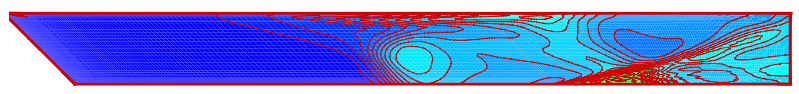

$C R=4$

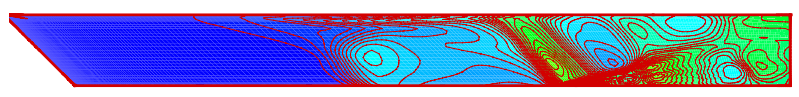

$C R=6$

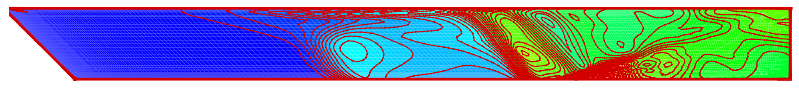

$C R=8.5$

Fig. $12 P / P_{0}$ contours on symmetry plane

For $C R$ of 8.5, as shown in Fig. 11 (a), and 11 (b), the pressure rise in the throat is up to a compression of 16. This excessive backpressure has the probability to choke the internal flow. The salient feature is also seen in Fig. 11 (c), a pressure rise is evident from $X / L=0.9$ till the throat entrance. It reveals that the shock wave is pushed away from the cowl lip, and propagates upstream of the throat. Furthermore, Fig. 12 indicates that most airflow is choked at the throat entrance, and then disgorges outward resulting in the strong spillage flow. As a result, this inlet is potentially unstarted, that is, under the computational condition $C R$ of 8.5 is conservatively proved to be unstart. Therefore, the visible reason for the unstart phenomena is perhaps the disgorgement of a shock wave system upstream of the cowl lip. The unstart inlet relates directly to an excessive spillage flow as a result of too great $C R$. In general, during the design and optimization of inlet configurations, the inlet should make the best of the incoming airflow with the utmost compression.

\subsubsection{Performance Parameters}

The significant quantities of mass capture ratio $m_{c} / m_{0}$ and compression ratio $P_{t h} / P_{0}$ generally represent the inlet performance of capturing the inflow and compression the captured flow, respectively. Besides the two, several nondimensional terms are used as performance parameters to assess the operation characteristics as follows: throat Mach number $M a_{t h}$, total pressure recovery $P_{t, t h} / P_{t, o}$, and kinetic energy efficiency $\eta_{K E}$.

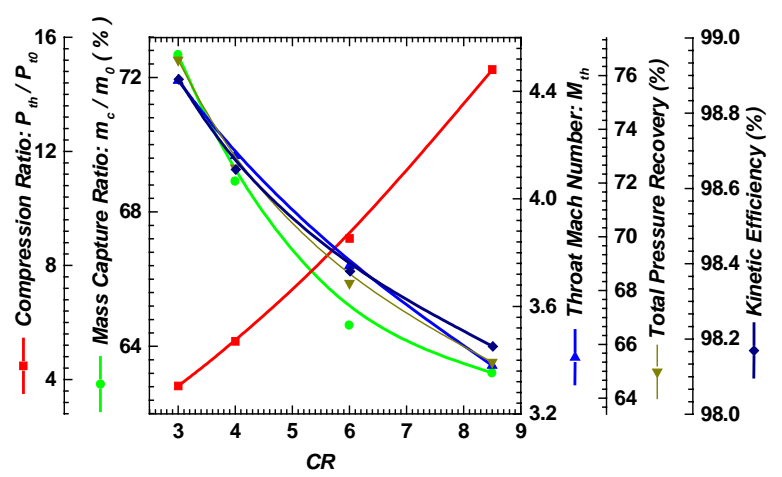

Fig. $13 C R$ effects on performance parameters

Fig. 13 demonstrates $C R$ effects on the inlet performance parameters. It appears that as $C R$ is gradually increased from 3 to 8.5 , the compression ratio rises from 3.77 to 14.86 , and the mass capture ratio declines from $72.67 \%$ to $63.21 \%$. The trends is excellently captured in the former discussion, and indicate that higher $C R$ induces more reflected shock waves inside the inlet, thus incrementally increasing the compression ratio and airflow downward angle. As a result, more airflow disgorges out of the inlet, and then increases the flow spillage.

Over the wide range of $C R$, throat Mach number, total pressure recovery, and kinetic energy efficiency are also shown in Fig. 13. While increasing $C R$, throat Mach number is observed to decrease from 4.44 to 3.38. And then, total pressure recovery and kinetic energy efficiency decrease from $72.57 \%$ and $98.89 \%$ to $65.33 \%$ and $98.18 \%$, respectively with the similar tendency. This can also primarily attributed to the increase of reflected shock waves due to the increase of $C R$. Herein it is noteworthy that the spline 
approximation curves given in the figure are useful to quantitatively predict the correlations between $C R$ and the performance parameters.

\section{Conclusions}

In conclusion, the salient features obtained from the study may be summarized as follows:

(1) The CFD results show favorable agreement with the experimental data, and reveal the detail flow characteristics of the inner and outer flow fields.

(2) Under the computational condition, the inlet of contraction ratio 8.5 is potentially unstarted due to the disgorged strong shock wave upstream of the throat station.

(3) As the contraction ratio gradually increases, an increase in compression ratio, and decreases in mass capture ratio, kinetic energy efficiency, total pressure recovery, and throat Mach number are excellently captured. Therefore, certain recommendations for the improvements of inlet performance can be provided to design and optimize the scramjet inlet configurations.

\section{ACKNOWLEDGEMENTS}

Funding for this study was jointly provided by Chinese Academy of Sciences and National High Technology Program.

\section{REFERENCES}

1. Trexler, C. A.: Inlet Performance of the Integrated Langley Scramjet Module (Mach 2.3 to 7.6), AIAA/SAE $11^{\text {th }}$ Propulsion Conference, September 29-October 1, 1975, Anaheim, CA, AIAA-75-1212.

2. Kanda, T.; Komuro, T.; Masuya, G.; Kudo, K.; and Murakami, A.: Mach 4 Testing of Scramjet Inlet Models, AIAA-89-2680, July 1989.

3. Srinivasan, S.; McClinton, C. R.; and Kamath, P. S.: Numerical Simulation of Flow Through the Langley Parametric Scramjet Engine, SAE Tech. Paper Ser. 892314, September 1989.

4. Holland, S. D.; and Perkins, J. N.: Mach 6 Testing of Two Generic Three-Dimensional Sidewall Compression Scramjet Inlets in Tetrafluoromethane, AIAA-90-0530, January 1990.

5. Zhang, K.; Xiao, X.; and Xu, H.: The Parametric Analysis and Experimental Investigation of Sidewall Compression Inlets at Mach 5.3 in Non-Uniform Incoming Flow Condition, AIAA-95-2889, July, 1995.

-6. Kubota, H.; and Stollery, J. L.: An Experimental Study of the Interaction Between a Glancing Shock Wave and a Turbulent Boundary Layer, J. Fluid Mech., Vol. 116, 1982, pp. 431-458.

7. Settles, G. S.; and Dolling, D. S.: Swept Shock/Boundary-Layer Interactions-Tutorial and Update, AIAA-90-0375, January 1990.

8. Knight, D. D.; Horstman, C. C.; and Settles, G. S.: Three-Dimensional Shock Wave-Turbulent Boundary Layer Interactions Generated by a Sharp Fin at Mach 4, AIAA-92- 0648, Jan. 1991.

9. Panaras, A. G.; and Stanewsky, E.: Numerical Study of Secondary Separation in Glancing Shock/Turbulent Boundary Layer Interactions, AIAA-92-3666, July 1992.

10. Settles, G. S.; and Dolling, D. S.: Swept Shock-Wave/Boundary-Layer Interactions, Tactical Missile Aerodynamics - General Topics, AIAA J., 1992, pp. 505-574.

11. Holland, S. D.; and Perkins, J. N.: Internal Shock Interactions in Propulsion/Airframe Integrated Three-Dimensional Sidewall Compression Scramjet Inlets, AIAA-92-3099, July, 1992.

12. Engineering Sciences, Inc.: UNIC CFD Code Fluid Dynamics Modeling for Engineering fluid and Thermal Analyses.

13. Chang, X.; Chen, L.; Yu, G.; and Qian, D.: Development of a Facility for Model Scramjet Testing, AIAA-2001-1857, 2001.

14. Trexler, C. A.; Auslender, A. H.; Weidner, J. P.: Experimental Investigation of Inlet-Combustor Isolators for a Dual-Mode Scramjet at a Mach Number of 4, NASA TP-3502, 1995. 